\title{
The Space Technology 5 Power System Design
}

\author{
Karen D. Stewart ${ }^{*}$ and Amri I. Hernandez-Pellerano. ${ }^{\dagger}$ \\ NASA/Goddard Space Flight Center, Greenbelt, MD, 20771
}

\begin{abstract}
The Space Technology 5 (ST5) mission is a NASA New Millennium Program (NMP) project that was developed to validate new technologies for future missions and to demonstrate the feasibility of building and launching multiple, miniature spacecraft that can operate as science probes, collecting research quality measurements. The three satellites in the ST5 constellation will be launched into a sun synchronous LEO (Low Earth Orbit) in early 2006. ST5 fits in the 25 kilogram and 24 Watt class of miniature but fully capable spacecraft. The power system design features the use of new technology components and a low voltage power bus. In order to hold the mass and volume low and to qualify new technologies for future use in space, high efficiency triple junction solar cells and a lithium ion battery were baselined into the design. The Power System Electronics (PSE) was designed for a high radiation environment and uses hybrid microcircuits for power switching and over current protection. The ST5 power system architecture and technologies will be presented.
\end{abstract}

\section{Introduction}

$\mathrm{T}$ HE goal of NASA's Space Technology 5 (ST5) mission include qualifying multiple new technologies for future use in space, building a miniature but full service spacecraft, taking localized magnetic field measurements for mapping purposes, and operating as a constellation of satellites. The New Millennium Program offers a unique opportunity to validate new technology components for future space flight use. The small, low cost Space Technology 5 (ST5) platform provides a means by which these new technologies can gain flight qualification and flight heritage status without posing a risk to higher cost science missions. The new technologies that have been space qualified for ST5 are the lithium ion battery, triple junction solar cells, X-band digital transponder, miniature spinning sun sensor, cold gas micro thruster, CULPRiT (CMOS Ultra Low Power Radiation Tolerant) logic, X-band evolved antenna, thermal variable emittance coatings and miniature science grade magnetometer.

Another goal of the ST5 mission is to produce a miniature but fully capable scientific spacecraft. ST5 is considered the first step in a goal towards a further reduction in size to a nanosat-sized platform that may be used on future missions. Such missions would deploy multiple miniature spacecraft as science probes to conduct localized measurements on orbit. In order to meet these goals, the volume, mass and power of all components had to shrink significantly.

The ST5 mission is also tasked with operating multiple spacecraft as a constellation. This includes flight operations as well as developing and testing three spacecraft at once, sharing and juggling the resources of the same team members and test equipment as well as gaining valuable experience managing efficiencies in the scheduling and budgeting of numerous parallel builds. The lessons learned from this program will be applied to future constellation missions.

\section{ST5 Spacecraft Overview}

The ST5 design emphasizes simplicity and reduction in scale. In order to simplify the guidance and navigation, the spacecraft was designed to be spin stabilized. The structure is octagonal, with 8 body-mounted solar panels. The top and bottom decks are removable to allow for assembly, integration and testing of the tightly packed components. The avionics on each satellite are combined into one unit. The Control and Data Handling unit $(C \& D H)$ is housed on a very densely populated double-sided card with approximately 2000 parts per side. The Power System Electronics (PSE) is located in the same enclosure with its interface with the C\&DH being their common backplane. This combined avionics box is called the Card Cage Assembly (CCA) as it also functions as the

\footnotetext{
* Power System Lead Engineer, Power Systems Branch, Code 563.

${ }^{\dagger}$ Power System Design Engineer, Power Systems Branch, Code 563.
} 
main structural element for the spacecraft. The CCA is responsible for processing all signals and power to and from each component on the spacecraft, and is, in essence, both the heart and brain of the craft. In order to meet the stringent magnetic field requirements, extra care was taken to minimize current loops and the CCA card layouts were scrutinized for such. Power and signals from the CCA to each component were combined in one connector per component in order to direct currents to return back to the source in the same harness bundle. The electrical isolation of the components was controlled in order to minimize ground currents in the metal structure.



Figure 1. Space View of the ST5 spacecraft

ST5's other new technology components include: a single-card Command and Data Handling (C\&DH) computer, a miniature communications system featuring an X-band transponder, a cold gas propulsion system using a single micro-thruster for both delta- $\mathrm{V}$ and attitude control, a miniature magnetometer, a miniature spinning sun sensor, and a "plug -and-play" ground system architecture. One of the new technology components is a miniature transponder that offers a substantial decrease in weight, power, and volume over current operational systems. Two different types of antennas are being developed for ST5: a quadrifilar helix antenna and an "evolved" antenna designed with genetic algorithms. A single cold-gas micro-thruster provides all ST5 orbit and attitude maneuver capability. A miniature digital sun sensor is used to measure the elevation angle of the sun with respect to the ST-5 spin axis.

\section{ST5 Power System Design}

There are a number of challenges imposed by the ST5 mission. Specifically, designing a nanosat class spacecraft demands that minimizing mass, volume and power dissipation drive the design. The result is a very streamlined approach, while striving to maintain a high level of capability. The tough radiation requirements, along with the low voltage bus, limit the parts selection of analog parts that operate within these constraints. The challenge of qualifying new technology components for the space environment in a short development schedule is another hurdle. The mission requirements also demand magnetic cleanliness in order to reduce the effect of stray magnetic fields on the science grade magnetometer.

The ST5 power system design features the use of new technology power system components while being constrained by the resources of mass, power and volume. In order to hold the mass and volume low and to qualify new technologies for future use in space, high efficiency triple junction solar cells and a lithium ion battery. were designed into the system. These high efficiency (28.5\%) triple junction solar cells and the 7.5 Amp-hour LithiumIon battery help to keep the mass of the system low while maximizing available power.

The main functions of the power system are to convert solar energy with the eight solar array panels, store any excess solar energy in the lithium-ion battery, draw stored energy from the battery when needed, and provide switched power outputs (with on/off control) to the S/C subsystems. Some of the key elements of the power system architecture are the low voltage busses $(+5.25 \mathrm{~V}$ regulated and $+7.2 \pm 1.2 \mathrm{~V}$ unregulated) along with components that achieve very low power consumption and dissipation. The low voltage busses were selected in order to minimize conversion and regulation losses. Since most of the spacecraft electronics (about 90\%) are digital and operate from 
an input voltage of $+5 \mathrm{~V}$, and since the components are in close proximity (less than 12 inches), a +5 Volt power bus was chogen. A higher voltage bus $(+7.2 \mathrm{~V} \pm 1.2 \mathrm{~V})$ was added for conponents that can improve efficiency when operated from a higher voltage (transponder high power ampliffer) or for noisier components that require filters and regulators at their input (spacecraft computer).

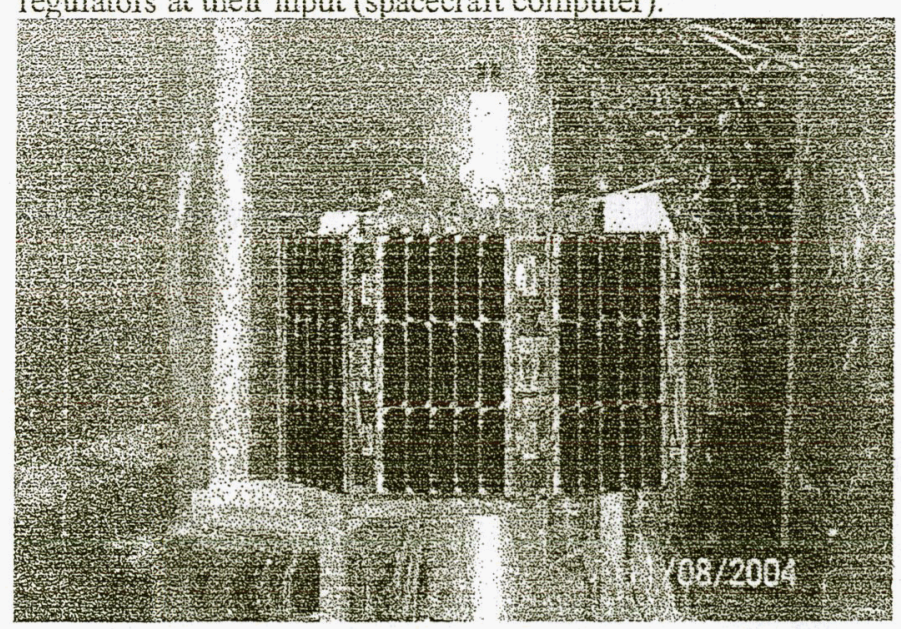

Figure 2. Photo of first spacecraft prior to close out work and start of environmental testing.

The ST5 power system is a direct energy transfer (DET) topology, battery bus design. This architecture was chosen for this miniature spacecraft because of its simplicity and small parts count. We tried to eliminate any extraneous parts in order to minimize dissipation and improve efficiency. Every diode drop in this low voltage bus design represented a potential loss of a large percentage of the nominal bus voltage compared to that for a $28 \mathrm{~V}$ system. For instance, a radiation hardened diode with a forward voltage drop of $0.38 \mathrm{~V}$ represents $5 \%$ of our low voltage bus compared te $1 \%$ of a $28 \mathrm{~V}$ bus. Therefore, the use and selection of each part in the primary power path were serutinized.

\section{Power System Electronics}

To meet the goals of producing a miniature power system and improving its efficiency, some functions of the Power System Electronics (PSE) were combined and streamlined. Combining the solar array regulator and battery charger into one low drop out voltage regulator also resulted in increasing reliability by minimizing the number of parts in the main power path. The dual role of this circuitry is performed by a pulse width modulated boost converter which regulates to a fixed voltage clamp when the battery is charged. The voltage clamp is set for the optimum end-of-charge voltage of the lithium-ion battery and provides overcharge protection. Battery overdischarge protection is provided by the flight software resident in the spacecraft computer. Analog battery health and status signals are digitized and monitored by the flight software. If the battery falls below a predetermined depth of discharge or voltage, a flight software load shedding algorithm is initiated to reduce demand on the battery.

As described previously, the PSE maintains an unregulated $+7.2 \pm 1.2 \mathrm{~V}$ primary power bus during battery charging and a regulated $+5.25 \mathrm{~V}$ secondary power bus. These two low voltage busses are shown in the simplified power system block diagram (Figure 3). Power distribution is accomplished with 10 digitally commandable and resetable solid state power switches. Each power switch has a digital command and status interface as well as an over-current protection circuit integrated into a small package. A circuit breaker function is activated when an overcurrent condition is sensed. The current level for the over-current trip function is adjustable for each load and is fixed prior to launch. The default state of a switch (after initial start-up) can be either on or off. Status bits indicate whether the switch has tripped due to over current and what state the switch has been commanded to This hybrid microcircuit is a custom Goddard design and has passed flight qualification testing.

As shown in Fig. 3 , there are five switchable $+7.2 \mathrm{~V}$ unregulated outputs. The $+7.2 \mathrm{~V}$ power to the transponder's $\mathrm{X}$-band uplink receiver is unswitched; it is always powered-on when the spacecraft is powered-on. A DC-to-DC converter provides regulated $+5.0 \mathrm{~V}$ power to the flight computer $(\mathrm{C} \& \mathrm{DH}$ board). The $+5.0 \mathrm{~V}$ power to the $\mathrm{C} \& \mathrm{DH}$ is always on, but it can be momentarily power cycled by a ground-to-transponder "special command" if necessary. At the bottom of the figure are the four switchable $+5.25 \mathrm{~V}$ regulated outputs. All of the PSE board's solid-state power switches are controlled by the C\&DH processor via read/write transactions over the backplane's local 16-bit parallel 
data bus. Some other functions of the power system electronics include analog signal processing. Signals from voltage, current and temperature sensors are processed and multiplexed for power system health and status monitoring.

The PSE provides a dedicated switching converter for the $\mathrm{C} \& \mathrm{DH}+5 \mathrm{~V}$ service. Initially, a commercial off-theshelf switching converter was chosen for this purpose whose input voltage range was low enough to support a possible $6-8 \mathrm{~V}$ allowable span. However, this package was susceptible to radiation and was noisy even with added filtering, so a discrete design was produced. Although the discrete converter requires more board space, it is over $90 \%$ efficient, radiation-tolerant to over 100 krads total dose, immune to major single event effects and achieves a low noise output. The design tradeoff in this case was to choose the improvement in performance at the cost of a bit more board space.

Early in the design phase, some of the tough challenges involved finding analog parts that could meet the 100 $\mathrm{krad}$ total dose radiation requirement in addition to the low supply voltage of $5 \mathrm{~V}$. For example, most of the analogto-digital (A/D) converters and op amps that could operate from the low supply voltage of $5 \mathrm{~V}$ were not sufficiently radiation hardened. We were not able to use the majority of parts from other missions due to the low voltage requirement for these analog parts. Eventually, we gathered enough data from radiation testing and breadboard experience to select analog parts that could operate well from the low voltage and meet the performance requirements during the anticipated radiation exposure.

Having initially been designed as a secondary payload, the ST5 spacecraft was designed to be powered off during launch and ascent. Upon separation from the launch vehicle, a power system enable switch will close and the spacecraft will turn on. For integration and test (I\&T) purposes there is a battery off-line switch that prevents even small amounts of leakage from the battery when the $\mathrm{S} / \mathrm{C}$ is powered off for extended periods of time. Also for I\&T, the PSE has an externally accessible connector for supplying ground power, charging the battery, and directly measuring the power system's health and status signals.

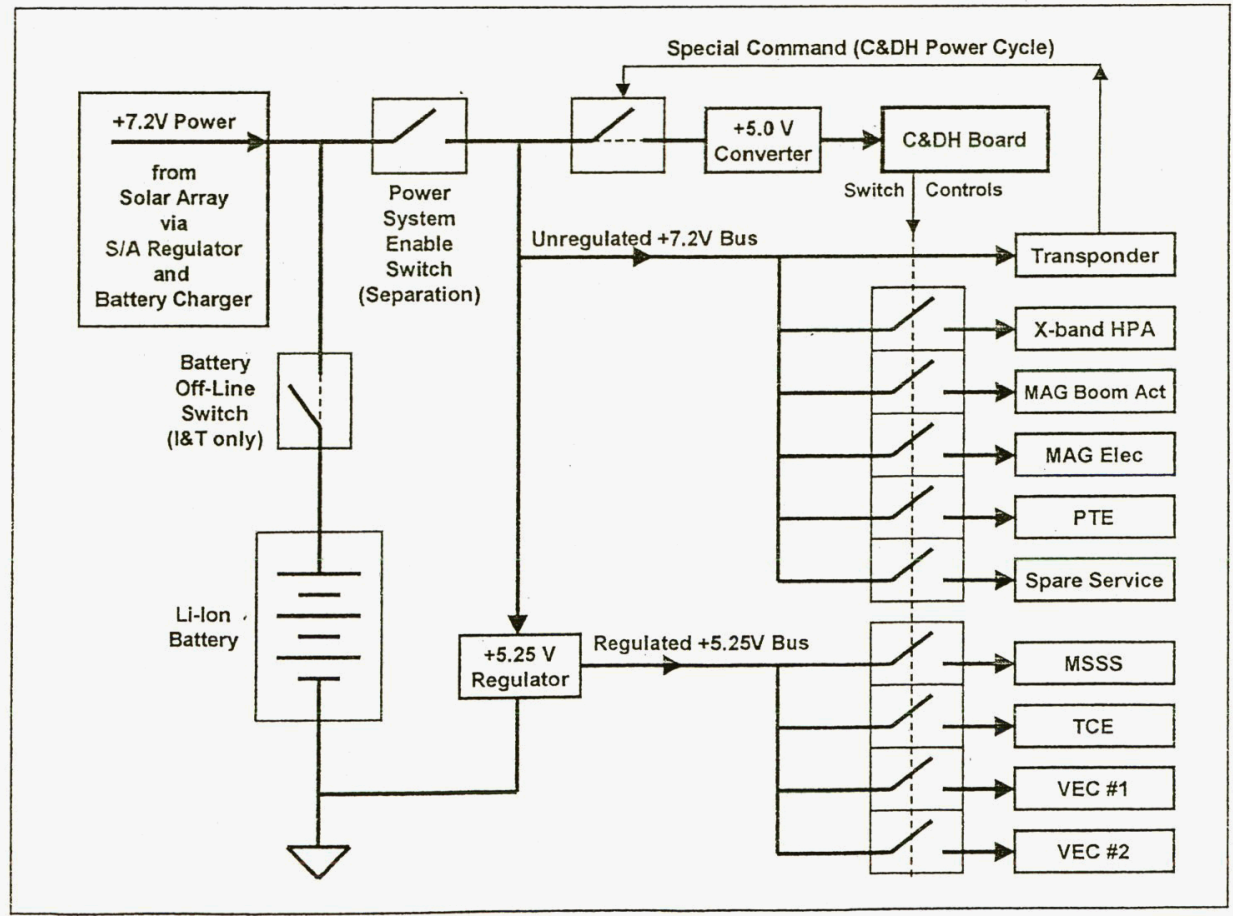

Figure 3. Simplified Distribution Diagram of the ST5 Power System Electronics 




\section{Figure 4. Power System Electronics (PSE) Card}

Reducing the mass, volume and power consumption of the power system was a fundamental design goal for this system. The entire PSE board mass is approximately $2.1 \mathrm{~kg}$, ineluding all card stiffeners, its aluminum core and heatsink. There is some mass penalty inherited by the PSE owing to the fact that this card is used as a structural element for the spacecraft. The PSE could have been packaged in a smaller and lighter weight fashion, however the system level mass savings gained by the multiple functions outweighed that option. Figure 4 is a photograph of the A side of the PSE board assembly. The total power consumption of the card is about 0.9 watts, including all regulators and converters. The entire PSE dissipates between 1.9 and 2.6 Watts, depending on the spacecraft load and state of charge of the battery.

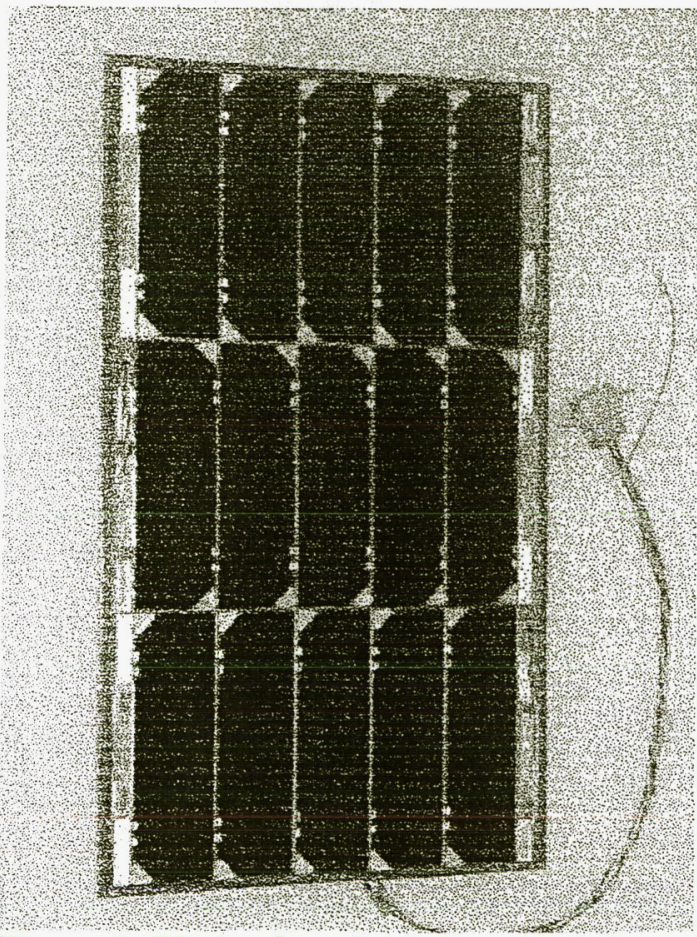

Figure 5. Photo of one ST5 solar panel.

\section{Solar Arrays}

The ST5 solar array is comprised of eight body-mounted panels. The solar cells are triple-junction GaAs (InGaP/InGaAs/Ge) cells and have an average cell efficiency of $28.5 \%$, with a maximum efficiency greater than $29 \%$. At beginning of life, power generation by the solar array will be just over 26 watts. Emcore fabricated the cells, then assembled and tested the panels. The solar panels were designed to be identical, thus enabling spare panels to substitute at any panel location. This also made the fabrication simpler and reduced the risk of errors during the development and integration stages. Temperature sensors are monitored on two of the eight panels.

The strict magnetic cleanliness requirement was tackled by mirroring the current in the solar cell strings with a similar ground path underneath. This was accomplished by providing the return current a path composed of a flat wire mesh that was installed on the front side of the panel substrates before the cell strings are laid down. This method was found to have better performance than conventional backwiring with a modest cost impact.

Due to growth in the power load demand of the spacecraft, in addition to the limited solar panel area available around the sides of a small vehicle, the battery will be used to supplement the solar arrays during peak load conditions in sunlight. Periods of peak power demand are expected to be during $\mathrm{X}$-band downlink passes when the HPA (High Power Amplifier) is on. Active power management will be done by the ground system to monitor and adjust power use. The battery also powers the spacecraft during eclipses. 


\section{Batteries}

ST5 has a single flight battery which provides power for peak loads and during eclipses. Considered a new technology on the ST5 mission, the 7.5 Amp-hour Lithium-Ion battery has completed a successful flight qualification program. Lithium-Ion was the battery technology chosen due to its high energy density and promising performance. The ST5 battery is comprised of 12 Sony 18650 high reliability lithium ion cells. The cells are arranged in 6 strings of 2 series connected cells. The individual battery cells have an end-of-charge voltage of $+4.2 \mathrm{~V}$ each, with a capacity of about 1.25 amp-hours at a $\mathrm{C} / 5$ discharge rate, to a voltage of $+3.0 \mathrm{~V}$. The total battery voltage is $+8.4 \mathrm{~V}$ maximum and the total battery capacity is approximately 7.5 Amp-hours at a rate of $\mathrm{C} / 5$. Figure 6 is a picture of the battery, with a one inch scale in the foreground. AEA Technology Space in England performed the battery detailed design, assembly and qualification testing.

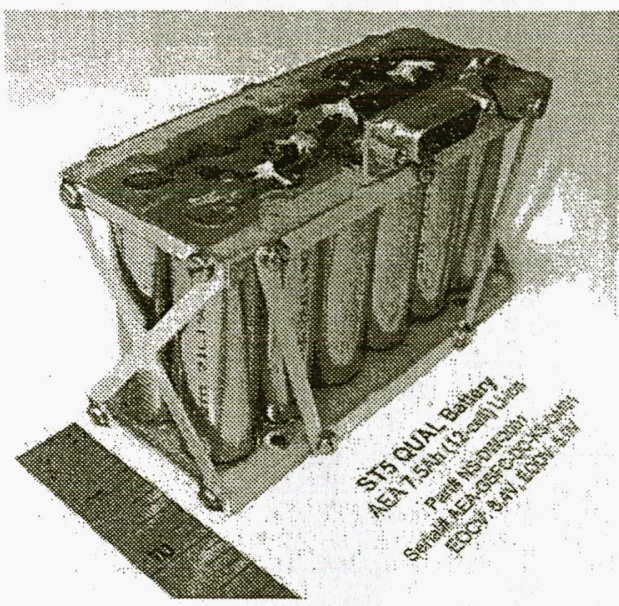

Figure 6: Photograph of ST5 Lithium-Ion Battery

The ST5 lithium-ion battery provides for an. increase in energy density of 2-3 times over nickel cadmium batteries, thus helping our mass and volume allocations. Along with the increase in energy density, this battery technology allows for a simplification in battery charge management circuitry. Constant current charging is no longer required and the voltage and temperature management is greatly simplified. In addition, the selection of low amp-hour cells in the battery has allowed for further reduction of battery charge control circuitry compared to that required for larger lithium-ion cells. As a standard practice, the AEA (Sony 18650) cells go through lot acceptance testing, where they have been matched for cell performance of a number of parameters. Only the screened and matched cells are used in the battery. Additionally, the lower amp hour size cells are arranged in multiple strings, each sharing a portion of the full battery current, rather than one single string of cells taking the full charge and discharge current. In this ST5 arrangement, any individual cell imbalance will have less effect on the entire battery. Thus, the cell balancing electronics needed for larger cell sizes are not necessary for the ST5 cells. Here we are simply using battery level charge control measures.

Another advantage of the battery cells selected for ST5 is the inherent safety features. There is built in overcharge protection in each cell which removes the cell from charge under severely overcharged conditions. Having this mechanism internal to the cell alleviates the need for external electronics to bypass or clamp individual cells, and therefore simplifies the peripheral hardware typically associated with battery management of lithium ion cells. The reduction in the electronics area simplifies the design and testing and is consistent with our mass and volume constraints.

\section{Conclusion}

In addition to designing and developing a new spacecraft architecture for three spacecraft, multiple new technologies had to progress through a space flight qualification program at the same time on ST5. Having gained flight qualification status while in development for ST5, these new technologies have cleared a hurdle for potential future use on larger scientific missions. The lessons learned from the development and operation of a small constellation of satellites will be used on follow-on programs.

The ST5 mission is currently progressing through the final stages of the test program. All of the components are currently integrated and operating on the spacecraft. The first of the three spacecraft has successfully completed all environmental testing. The remaining two spacecraft in the ST5 constellation are being tested in parallel and are progressing through spacecraft level environmental testing. The scheduled launch for ST5 is in February 2006.

\section{Acknowledgments}

The authors would like to thank Roberto Arocho and Syed Ameen for their excellent support in the testing of the ST5 PSE cards as well as the integrated avionics box. 